\title{
Effect of intravenous amino acids on interdigestive antroduodenal motility and small bowel transit time
}

H A J Gielkens, A van den Biggelaar, J Vecht, W Onkenhout, C B H W Lamers, A A M Masclee

\begin{abstract}
Background-Patients on total parenteral nutrition have an increased risk of developing gallstones because of gall bladder hypomotility. High dose amino acids may prevent biliary stasis by stimulating gall bladder emptying.

Aims-To investigate whether intravenous amino acids also influence antroduodenal motility.

Methods-Eight healthy volunteers received, on three separate occasions, intravenous saline (control), low dose amino acids (LDA), or high dose amino acids (HDA). Antroduodenal motility was recorded by perfusion manometry and duodenocaecal transit time (DCTT) using the lactulose breath hydrogen test.

Results-DCTT was significantly prolonged during LDA and HDA treatment compared with control. The interdigestive motor pattern was maintained and migrating motor complex (MMC) cycle length was significantly reduced during HDA compared with control and LDA due to a significant reduction in phase II duration. Significantly fewer phase IIIs originated in the gastric antrum during LDA and HDA compared with control. Duodenal phase II motility index was significantly reduced during HDA, but not during LDA, compared with control. Conclusions-Separate intravenous infusion of high doses of amino acids in healthy volunteers: (1) modulates interdigestive antroduodenal motility; (2) shortens MMC cycle length due to a reduced duration of phase II with a lower contractile incidence both in the antrum and duodenum (phase I remains unchanged whereas the effect on phase III is diverse: in the antrum phase III is suppressed and in the duodenum the frequency is increased); and (3) prolongs interdigestive DCTT.
\end{abstract}

(Gut 1999;44:240-245)

Keywords: amino acids; antroduodenal motility; small bowel transit time; total parenteral nutrition

Patients receiving total parenteral nutrition (TPN) have an increased risk of developing gall bladder sludge and stones. ${ }^{1-3}$ Bile stasis in the gall bladder during prolonged TPN is the major risk factor in the development of gall stones in these patients. ${ }^{4}$ The occurrence of gall bladder disease during TPN is prevented by intermittent intravenous injections of cholecystokinin (CCK), leading to profound gall bladder contraction. ${ }^{6}{ }^{7}$ However, recent studies have shown that separate intravenous infusion of amino acids at high doses also induces gall bladder emptying. ${ }^{8-10}$ It has been suggested that this may be of clinical relevance as an alternative means, by modification of the nutrient regimen, of preventing TPN associated cholelithiasis. ${ }^{8-10}$

In addition to inducing gall bladder emptying, intravenous amino acids stimulate gastric acid secretion, ${ }^{11}{ }^{12}$ may increase pancreatic enzyme secretion, ${ }^{13}$ and delay gastric emptying of liquids. ${ }^{14}$ However, little is known about the possible effects of separate intravenous infusion of high doses of amino acids on gastrointestinal motility. Alterations in gastrointestinal motility may influence the risk of developing gall bladder sludge and stones: slow intestinal transit increases the risk of gallstone formation by influencing biliary lipid composition. ${ }^{15}{ }^{16} \mathrm{We}$ have studied the effect of two different doses of a commercially available mixed amino acids solution on interdigestive antroduodenal motility and small bowel transit time in healthy volunteers.

\section{Methods}

SUBJECTS

Eight healthy volunteers (five men, three women; mean age 23 years, range 20-34 years) participated in the study. None of the subjects had a history of gastrointestinal disease or surgery and none was taking any medication. Informed consent was obtained from each individual and the protocol was approved by the ethics committee.

STUDY PROTOCOL

Each subject participated in three experiments performed on separate days in random order with an interval of at least seven days during continuous infusion of either saline (control) or amino acids (Vamin 18EF, Kabi Pharmacia BV, Woerden, The Netherlands), given at a low dose (125 mg protein $/ \mathrm{kg} / \mathrm{h} ; \mathrm{LDA})$ and at a high dose (250 mg protein $/ \mathrm{kg} / \mathrm{h}$; HDA). The experiments were started at 800 am after an overnight fast. The manometric assembly was

Abbreviations used in this paper: $\mathrm{CCK}$,

cholecystokinin; DCTT, duodenocaecal transit time; HDA, high dose amino acids; LDA, low dose amino acids; MMC, migrating motor complex; TPN, total parenteral nutrition; NO, nitric oxide. 
positioned as described below. Two intravenous cannulas - one for blood sampling, the other for infusion-were inserted into the antecubital veins of each arm. The subjects were studied in a semirecline position. Antroduodenal motility was recorded continuously. Fifteen minutes after the onset of spontaneous phase III motor activity in the proximal duodenum, infusion of either saline or amino acids was started (defined as time 15 minutes) and continued for 375 minutes. At time 0 minutes, $6 \mathrm{~g}$ of lactulose (Legendal, Inpharzam, Amersfoort, The Netherlands), dissolved in $60 \mathrm{ml}$ distilled water (osmolality $292 \mathrm{mmol}$ ) was administered slowly over 10 minutes into the distal duodenum through the central lumen of the manometric assembly in order to determine duodenocaecal transit time. Antroduodenal motility was recorded continuously; breath samples were obtained at 10 minute intervals. Blood samples for measurement of plasma CCK concentrations were obtained at times $-30,-15,0,15,30,45,60$, $90,120,180,240,300$, and 360 minutes. In addition, blood samples for measurement of plasma amino acid concentrations were obtained at time -15 minutes and at time 120 minutes.

\section{ANTRODUODENAL MANOMETRY}

Antroduodenal pressures were recorded using a water perfused stationary manometry system. A polyvinyl multilumen tube of $6 \mathrm{~mm}$ outer diameter incorporating six catheters with side holes located at $0,5,10,15,25$, and $30 \mathrm{~cm}$ from the distal tip (Arndorfer Medical Systems, Greendale, Wisconsin, USA) was introduced transnasally and placed into the duodenum using a guide wire. Correct positioning of each of the pressure ports, the two side holes in the antrum and the four in the duodenum, was verified by fluoroscopy. The distal tip of the tube was located near the ligament of Treitz. Each lumen was connected to a pressure transducer (Medex, Hilliard, Ohio, USA) and perfused with gas-free distilled water by a low compliance pneumohydraulic infusion pump (Arndorfer Medical Supplies, Greendale, Wisconsin, USA) at a rate of $0.5 \mathrm{ml} / \mathrm{min}$. The outputs from the pressure transducers were processed by an eight channel polygraph (Synectics Medical, Stockholm, Sweden), displayed on a monitor screen and stored on a personal computer system pending analysis. At the end of each experiment correct positioning of the manometric assembly was again verified by fluoroscopy.

SMALL BOWEL TRANSIT TIME

Lactulose breath hydrogen analysis was used to measure duodenocaecal transit time, as described by Bond and Levitt. ${ }^{17}$ End expiratory breath samples were collected under basal conditions and every 10 minutes after the lactulose had been administered intraduodenally until a sustained rise in breath hydrogen excretion occurred. Breath samples were collected in 25 $\mathrm{ml}$ plastic syringes and analysed immediately using a hydrogen breath test unit (Lactoscreen, Hoekloos, Amsterdam, The Netherlands).
Duodenocaecal transit time was defined as the time between administration of lactulose (time 30 minutes) and a sustained rise in breath $\mathrm{H}_{2}$ concentration of at least 10 parts per million (ppm) over basal.

ASSAYS OF AMINO ACIDS AND CCK

Plasma levels of amino acids were measured as described previously. ${ }^{18}$ Plasma CCK was measured using a sensitive and specific radioimmunoassay using antibody T204. ${ }^{19}$

\section{ANALYSIS OF MOTILITY DATA}

Antroduodenal motility recordings were analysed both visually and by computer. The individual tracings were processed by specialised software (Polygram, Synectics Medical, Stockholm, Sweden) for adjusting baselines and extracting respiratory artefacts. However, the computer program does not recognise simultaneous pressure events as artefacts. Therefore, remaining artefacts obviously due to increases in intra-abdominal pressure were identified visually and excluded from analysis. Antral motility was analysed using the pressure tracings recorded from the side hole located most distal in the antrum $(25 \mathrm{~cm}$ from the distal tip); duodenal motor characteristics were analysed using the pressure tracings recorded from the side hole in the proximal duodenum (10 cm from the distal tip) and from the most distal side hole (at the tip). Antral phase III of the migrating motor complex (MMC) was defined as rhythmic contractile activity at maximum frequency (three contractions per minute) for at least one minute in temporal relation with duodenal phase III activity. Duodenal phases of the MMC were defined as follows: phase I, motor quiescence (no more than two contractions per 10 minutes) for at least five minutes and preceded by phase III; phase II, irregular contractile activity at a frequency of more than two contractions per 10 minutes; phase III, regular rhythmic contractile activity at a frequency of 10-12 contractions per minute for at least two minutes. Phase IIIs had to be propagated over at least two recording sites. MMC cycle length was defined as the time between the end of phase III in the proximal duodenum and the end of the next phase III. Origin (antrum versus duodenum), duration, and propagation velocity of each duodenal phase III were analysed visually. Propagation velocity was defined as the time interval between the onset of phase III activity in the proximal site and that in the distal site. Frequency, amplitude, and duration of the individual contractions were measured for both phase II and phase III using the computer program. Only pressure waves with an amplitude of at least $10 \mathrm{~mm} \mathrm{Hg}$ and duration at least 1.5 seconds were considered as true contractions. Additionally, motility indices of the distal antrum and the proximal duodenum were calculated as area under the contraction curves for the last 30 minutes preceding each duodenal phase III activity. 


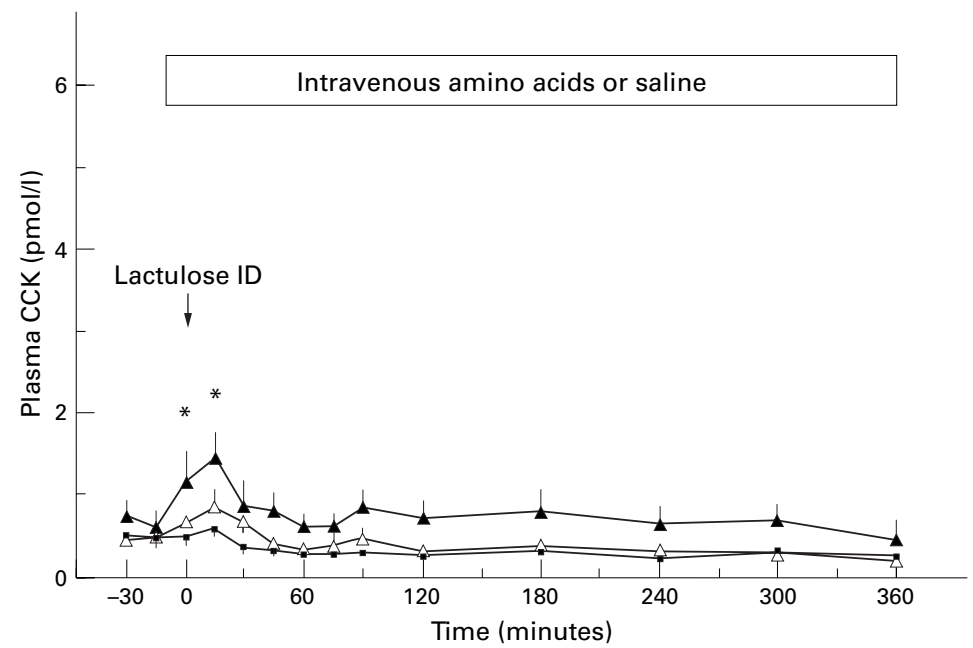

Figure 1 Plasma CCK concentrations (mean (SEM)) during LDA (open triangles) or $H D A$ (closed triangles) compared with control (squares). *Significantly different $(p<0.05)$ compared with basal.

STATISTICAL ANALYSIS

Results are expressed as mean (SEM). Differences in plasma CCK levels were analysed for statistical significance by multiple analysis of variance (MANOVA). Differences in plasma amino acid levels were analysed for statistical significance by paired analysis of variance. Data on phase III characteristics were pooled and analysed for statistical significance by unpaired analysis of variance. All other motility data were analysed for statistical significance by paired analysis of variance. Normal distribution of data was verified using the Levine test. When analysis of variance indicated a probability of less than 0.05 for the null hypothesis, Student-Newman-Keuls analyses were performed to determine which values between or within the experiments differed significantly. The significance level was set at $\mathrm{p}<0.05$.

Table 1 Plasma amino acid concentrations before (basal) and after LDA or HDA

\begin{tabular}{|c|c|c|c|c|}
\hline & Vamin 18EF (mmol/l) & Basal ( & $L D A(\mu m o l / l)$ & $H D A(\mu m o l / l)$ \\
\hline Alanine & 180 & $324(23)$ & $547(21)^{\star}$ & $690(34)^{\star}+$ \\
\hline Arginine & 54 & $115(11)$ & $227(19)^{\star}$ & $362(18)^{\star} \dagger$ \\
\hline Aspartic acid & 26 & $25(2)$ & $34(3)^{\star}$ & $44(4)^{\star} \dagger$ \\
\hline Glutamic acid & 33 & $152(26)$ & $167(27)$ & $180(20)$ \\
\hline Glycine & 75 & $218(22)$ & $339(24)^{\star}$ & $442(21)^{\star} \dagger$ \\
\hline Histidine & 44 & $77(2)$ & $140(5)^{\star}$ & $204(17)^{\star} \dagger$ \\
\hline Isoleucine & 43 & $84(7)$ & $163(9)^{\star}$ & $273(20)^{\star} \dagger$ \\
\hline Leucine & 60 & $136(11)$ & $215(10)^{\star}$ & $355(24)^{\star}+$ \\
\hline Methionine & 38 & $21(2)$ & $80(1)^{\star}$ & $150(13)^{\star} \dot{\dagger}$ \\
\hline Phenylalanine & 48 & $62(5)$ & $144(5)^{\star}$ & $245(22)^{\star} \dagger$ \\
\hline Proline & 59 & 229 (37) & $424(57)^{\star}$ & $580(37)^{\star}+$ \\
\hline Serine & 43 & $122(4)$ & $196(8)^{\star}$ & $255(14)^{\star} \dagger$ \\
\hline Threonine & 47 & $140(5)$ & $268(8)^{\star}$ & $354(24)^{\star}+$ \\
\hline Tryptophan & 9 & $56(6)$ & $83(7)^{\star}$ & $108(8)^{\star} \dagger$ \\
\hline Valine & 62 & $262(22)$ & $394(12)^{\star}$ & $579(31)^{\star} \dagger$ \\
\hline
\end{tabular}

Amino acid concentrations expressed as mean (SEM).

The composition of the infused mixed amino acids solution (Vamin 18EF) is given as reference. ${ }^{\star} \mathrm{p}<0.05$ compared with control; $\nmid \mathrm{p}<0.05$ compared with LDA.

Table 2 Characteristics of migrating motor complex (MMC) cycles while on LDA or HDA compared with control

\begin{tabular}{llll}
\hline & Control & LDA & HDA \\
\hline Number of complete MMC cycles & $2.4(0.3)$ & $3.4(0.5)$ & $4.8(0.7)^{\star} \dagger$ \\
MMC cycle length (minutes) & $137(12)$ & $117(16)$ & $76(7)^{\star} \dagger$ \\
Phase I (minutes) & $16(3)$ & $16(2)$ & $14(2)$ \\
Phase II (minutes) & $115(12)$ & $96(15)$ & $57(6)^{\star} \dagger$ \\
Phase III (minutes) & $6(1)$ & $5(0)$ & $4(0)$ \\
\hline
\end{tabular}

Results expressed as mean (SEM).

${ }^{\star} \mathrm{p}<0.05$ compared with control; $\uparrow \mathrm{p}<0.05$ compared with LDA.

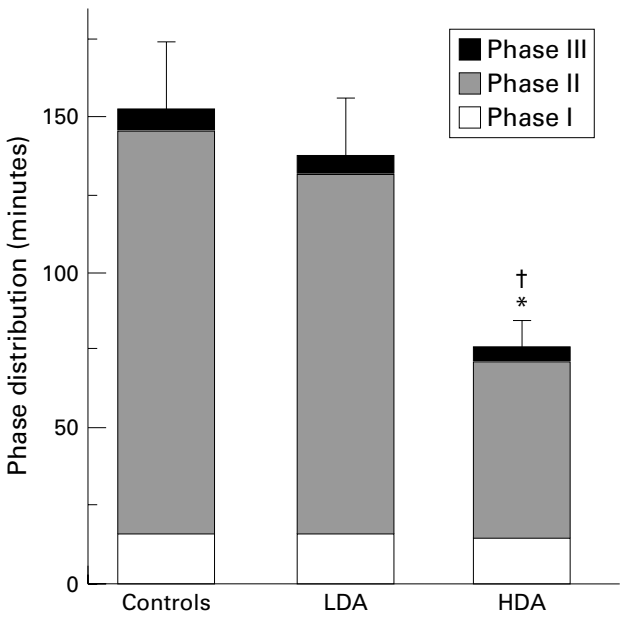

Figure 2 Data of mean MMC cycle length and its phase distribution (mean (SEM)) during LDA or HDA compared with control. * Significant $(p<0.05)$ difference compared with control; tsignificant $(p<0.05)$ difference between $L D A$ and $H D A$.

\section{Results}

PLASMA CCK

Plasma CCK concentrations at time -15 minutes were not significantly different among the three experiments (control: $0.5(0.1) \mathrm{pmol} / \mathrm{l}$; LDA: 0.5 (0.1) pmol/1; HDA: 0.6 (0.2) pmol/1). No significant changes in plasma CCK levels were observed during either control or LDA. However, during HDA plasma CCK levels showed a small but significant $(p<0.05)$ increase over basal levels during the first 30 minutes of infusion (fig 1).

PLASMA AMINO ACIDS

Table 1 presents plasma amino acid concentrations. No significant changes in plasma amino acid levels were observed in the control experiment. Intravenous infusion of amino acids dose dependently increased plasma levels of most amino acids, except glutamic acid.

ANTRODUODENAL MOTILITY

After the start of the intravenous infusion phase III recurred after 124 (34) minutes in the control experiment, after 114 (23) minutes in the LDA experiment, and after 53 (10) minutes in the HDA experiment $(\mathrm{p}<0.05$ versus LDA and control). A total of 19 complete MMC cycles were recorded during the control experiment, 27 during LDA and 38 during HDA. The number of complete MMC cycles recorded for each subject was significantly $(\mathrm{p}<0.05)$ higher during HDA (4.8 (0.7)) compared with control (2.4 (0.3)) and LDA (3.4 (0.5)). Individual mean MMC cycle length was significantly $(\mathrm{p}<0.05)$ reduced during HDA compared with control and LDA (table 2). The shorter duration of the MMC cycles during HDA resulted from a significant $(\mathrm{p}<0.05)$ reduction in phase II duration compared with both control and LDA (fig 2). During LDA individual mean MMC cycle length and phase II duration were not significantly different compared with control. Duration of phase I was not significantly different among the three experiments (table 2). 
Table 3 Characteristics of phase III of the migrating motor complex in the proximal duodenum while on $\mathrm{LDA}$ or $\mathrm{HDA}$ compared with control

\begin{tabular}{llll}
\hline & Control & LDA & HDA \\
\hline Antral origin/total & $8 / 19(37 \%)$ & $4 / 27(15 \%)$ & $3 / 38(8 \%)$ \\
Duration (s) & $321(23)$ & $285(17)$ & $260(11)^{\star}$ \\
Velocity (cm/min) & $15.1(2.3)$ & $16.5(3.2)$ & $11.5(1.5)$ \\
Frequency (contractions/min) & $11.3(0.1)$ & $11.4(0.1)$ & $11.1(0.2)$ \\
Mean amplitude (mm Hg) & $27.9(1.9)$ & $28.8(1.2)$ & $25.5(0.8)$ \\
Mean area under contractions (mm Hg.s) & $47.8(2.2)$ & $50.3(2.7)$ & $43.5(1.9)$
\end{tabular}

Results expressed as mean (SEM).

${ }^{\star} \mathrm{p}<0.05$ compared with control.

Table 4 Motility characteristics for the last 30 minutes of phase II preceding duodenal phase III activity of the distal antrum and proximal duodenum while on LDA or HDA compared with control

\begin{tabular}{llll}
\hline & Control & LDA & HDA \\
\hline Antrum & $0.39(0.10)$ & $0.26(0.09)$ & $0.09(0.03)^{\star}$ \\
Frequency (contractions/min) & $126(14)$ & $124(14)$ & $130(15)$ \\
Mean amplitude (mm Hg) & $5100(1585)$ & $3345(1669)$ & $881(335)^{\star}$ \\
$\quad$ Motility index (mm Hg.s) & & & \\
Duodenum & $1.00(0.17)$ & $0.81(0.11)$ & $0.56(0.08)^{\star}$ \\
$\quad$ Frequency (contractions/min) & $24.0(1.2)$ & $25.3(1.5)$ & $23.8(1.5)$ \\
Mean amplitude (mm Hg) & $1209(179)$ & $1025(137)$ & $699(141)^{\star}$ \\
Motility index (mm Hg.s) & &
\end{tabular}

Results expressed as mean (SEM).

${ }^{\star} \mathrm{p}<0.05$ compared with control.

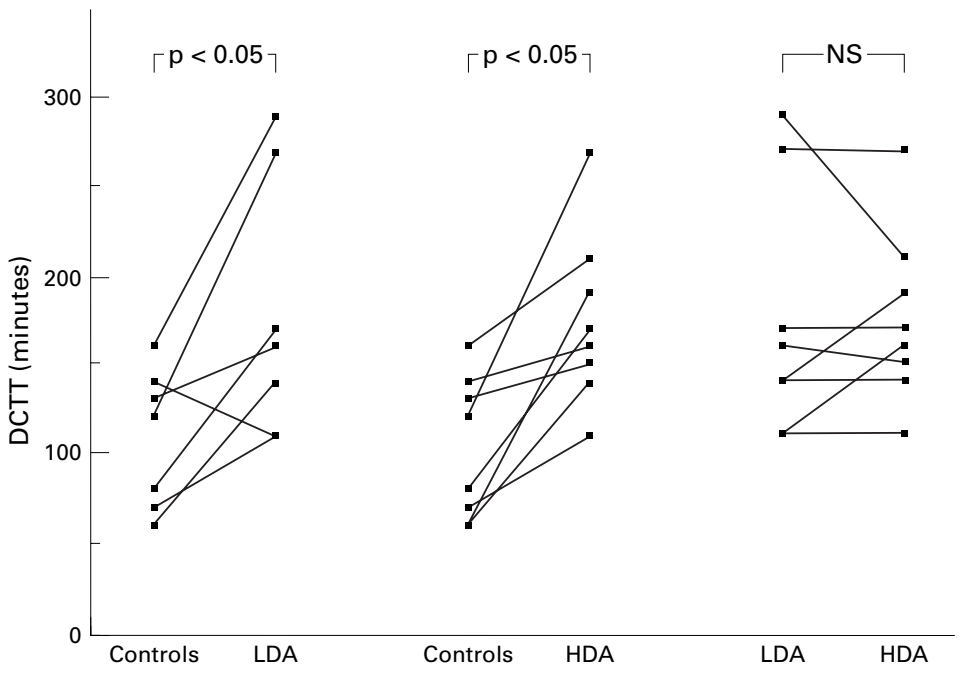

Figure 3 Individual data of duodenocaecal transit time (DCTT) during LDA or HDA compared with control.

Table 3 presents phase III characteristics of the proximal duodenum. During HDA phase III duration was significantly $(\mathrm{p}<0.05)$ shorter compared with control and its propagation velocity tended to be reduced. During both LDA and HDA significantly $(p<0.05)$ fewer phase IIIs originated in the gastric antrum.

Table 4 presents motility parameters of the distal antrum and the proximal duodenum for the last 30 minutes preceding duodenal phase III activity. Both antral and duodenal motility indices were significantly $(\mathrm{p}<0.05)$ reduced in late phase II during HDA compared with control due to significantly $(\mathrm{p}<0.05)$ reduced contraction frequency. No significant changes were observed during LDA, although antral motility tended to be reduced.

DUODENOCAECAL TRANSIT TIME

Figure 3 presents individual data for duodenocaecal transit time (DCTT). DCTT was significantly $(p<0.05)$ increased during both LDA (174 (24) minutes) and HDA (175 (17) minutes) compared with control (103 (14) minutes). DCTT was not significantly different between the low and the high dose intravenous amino acid infusions.

\section{Discussion}

The present study is the first to investigate the effects of separate intravenous infusions of amino acids on antroduodenal motility. Intravenous amino acids did not induce a fed-like motor pattern by disrupting the cyclic occurrence of the MMC. On the contrary, intravenous amino acids at high doses significantly increased duodenal phase III frequency. However, antral as well as duodenal phase II contractile activity was dose dependently reduced. Theoretically, the finding of a reduction in antral motility may result from technical shortcomings (e.g. catheter dislocation, number of antral recording sites). Correct positioning of the catheter, however, was checked before and after recording. Antral hypomotility was found only during HDA while the order of the experiments was randomised. Reduced antral motility may explain the delay in gastric emptying seen during infusion of intravenous amino acids. ${ }^{14} \mathrm{~A}$ recent study showed that intraduodenal L-tryptophan stimulates pyloric motility and may induce premature duodenal phase III activity. ${ }^{20}$ The present study shows that not only intraluminal but also intravenous amino acids affect antroduodenal motility.

Intravenous amino acids at both doses significantly prolonged DCTT despite increased duodenal phase III frequency. Luminal flow is known to be more rapid during phase III activity. ${ }^{21}$ A tendency towards a decreased propagation velocity of the phase III activity fronts was only seen during high dose intravenous amino acids and therefore does not offer a full explanation. However, physiological studies have shown that few phase IIIs eventually reach the caecum..$^{22}$ Perhaps propagation distances of phase III are reduced during intravenous amino acids but the present study design does not allow comment on this possibility as motility recordings of the small intestine were restricted to the duodenum. Transit of luminal contents also occurs during phase II, especially late phase II. ${ }^{21}{ }^{23}$ During high dose intravenous amino acids, duodenal phase II duration, the contraction frequency, and the motility index of late phase II were reduced. Therefore, intravenous amino acids may prolong intestinal transit time by reducing late phase II activity of the small intestine.

The mechanisms by which intravenous amino acids may affect gastrointestinal motility are largely unknown. Intravenous amino acids stimulate gastric acid secretion. ${ }^{112}$ Gastric acid inhibits antral phase III activity ${ }^{24}{ }^{25}$ and decreases MMC cycle frequency due to the increased duration of duodenal phase II. ${ }^{24}$ Therefore, the effect of intravenous amino acids on intestinal motility is probably not mediated by increased gastric acid secretion, but it may, at least partially, mediate the observed inhibition of antral motility. 
Intravenous amino acids induce gall bladder emptying $^{8-10}$ and may increase pancreatic enzyme secretion. ${ }^{13}$ The delivery of bile and pancreatic enzymes into the duodenum may affect gastrointestinal motility. Intraduodenal administration of pancreatic enzymes has no effect on interdigestive antroduodenal motility. ${ }^{26}$ Intraduodenal bile stimulates motilin release. ${ }^{27} 28$ In humans, intravenous infusion of motilin initiates antral phase III activity. ${ }^{29}$ However, a recent study showed that although duodenal perfusion of pancreaticobiliary juice during phase I of the MMC stimulates plasma motilin release it does not change MMC cycle length. ${ }^{30}$ Penagini and colleagues ${ }^{31}$ studied the effect of intrajejunal bile acids, infused at concentrations resembling postprandial levels, on interdigestive jejunal motility. Duration of the MMC and of its phases was not significantly altered, although there was a trend towards reduced duration of phase II during jejunal infusion of chenodeoxycholic acid. However, bile acids significantly reduced phase II contraction frequency and prolonged small bowel transit time. Therefore, the effect of intravenous amino acids on intestinal motility and transit may be partially mediated by increased intraluminal concentrations of bile acids as a result of significant gall bladder emptying.

In the present study intravenous infusion of amino acids at the high dose slightly but still significantly increased plasma CCK levels (0.5-1.5 pmol/1) for a short period of time. Infusion of CCK resulting in plasma CCK levels comparable with those seen after ingestion of a fatty meal (5-6 pmol/1) disrupts the MMC, inducing a fed-like motor pattern and accelerates small intestinal transit. ${ }^{32}$ Furthermore, loxiglumide, a CCK-A receptor antagonist, reduces meal stimulated antroduodenal motility and delays small intestinal transit, ${ }^{33}$ suggesting that CCK is involved in stimulatory regulation. Therefore, it seems unlikely that the effect of intravenous amino acids on antroduodenal motility and intestinal transit is mediated by CCK.

The mixed amino acids solution used in the present study contains about $11 \mathrm{~g} / 1 \mathrm{~L}$-arginine, a nitric oxide (NO) donor. Even when infused at a much higher rate than in the present study L-arginine has no effect on fasted antral motility in humans. ${ }^{34}$ In rats intravenous infusion of an NO donor during fasting disrupts the MMC and induces a fed-like motor pattern, whereas inhibition of NO synthase during feeding induces an MMC-like pattern. ${ }^{35}$ These data suggest that NO has no part in mediating the effect of intravenous amino acids on gastrointestinal motility.

Apart from L-arginine, commercially available mixed amino acids solutions contain other important amino acids. In the present study the intravenous amino acid mixture dose dependently increased plasma levels of many amino acids. The aromatic amino acids phenylalanine (Phe) and tryptophan (Trp) are potent stimuli of gastric acid secretion. ${ }^{112}$ It has been suggested that Phe and Trp may exert a direct effect on or near the parietal cell as parietal cell vagotomy does not inhibit their stimulatory effect on gastric acid secretion. ${ }^{12}$ Furthermore, in vitro studies in animals show that several amino acids (alanine, glycine, histidine, methionine, serine) are able to stimulate fundic or colonic smooth muscle directly. ${ }^{36}$ However, whether amino acids have any direct effect on human small intestinal smooth muscle in vivo is not known.

Single administration of high doses of intravenous amino acids may be used as a prophylactic against gallstones. One should be aware of the fact that intravenous amino acids, by significantly delaying intestinal transit, also influence biliary bile acid composition (increase in biliary deoxycholic acid) and thus may increase the risk of gallstone formation during TPN. ${ }^{15} 16$

In summary, the present study shows that in healthy volunteers high dose intravenous amino acids: (1) modulate interdigestive antroduodenal motility; (2) shorten MMC cycle length due to the reduced duration of phase II with a lower contractile incidence both in the antrum and duodenum (phase I remains unchanged whereas the effect on phase III is diverse: in the antrum phase III is suppressed and in the duodenum the frequency is increased); and (3) prolong interdigestive DCTT. The question arises whether these effects of high dose intravenous amino acids may interfere with the "housekeeper" function of the MMC.

Part of this work has been published in abstract form (Gastroenterology 1995;108:A726).

1 Messing B, Bories C, Kunstlinger F, et al. Does total parenteral nutrition induce gallbladder sludge formation and lithiasis? Gastroenterology 1983;84:1012-19.

2 Pitt HA, King W, Mann LL, et al. Increased risk of cholelithiasis with prolonged total parenteral nutrition. Am 7 Surg 1983;145:106-12.

3 Roslyn JJ, Pitt HA, Mann LL, et al. Gallbladder disease in patients on long-term parenteral nutrition. Gastroenterology 1983;84:148-54

4 Cano N, Cicero F, Ranieri F, et al. Ultrasonographic study of gallbladder motility during total parenteral nutrition. of gallbladder motility during to

5 Doty JE, Pitt HA, Porter-Fink V, et al. The effect of Doty JE, Pitt HA, Porter-Fink V, et al. The effect of
intravenous fat and total parenteral nutrition on biliary intravenous fat and total parenteral nutrition

6 Doty JE, Pitt HA, Porter-Fink V, et al. Cholecystokinin prophylaxis of parenteral nutrition-induced gallbladder disease. Ann Surg 1985;201:76-80.

7 Sitzmann JV, Pitt HA, Steinborn PA, et al. Cholecystokinin prevents parenteral nutrition induced biliary sludge in humans. Surg Gynaecol Obstet 1990;170:25-31.

8 Nealon WH, Upp JR Jr, Alexander RW, et al. Intravenous amino acids stimulate human gallbladder emptying and hormone release. Am f Physiol 1990;259:G173-8.

9 Zoli G, Ballinger A, Healy J, et al. Promotion of gallbladder emptying by intravenous aminoacids. Lancet 1993;341: $1240-1$

10 de Boer SY, Masclee AMM, Lam WF, et al. Effect of intravenous glucose on intravenous amino acid-induced gallvenous glucose on intravenous amino acid-induced gall39:268-74.

11 Isenberg JI, Maxwell V. Intravenous infusion of amino acids stimulates gastric acid secretion in man. $N$ Engl $\mathcal{F}$ Med 1978;298:27-9

12 McArthur KE, Isenberg JI, Hogan DL, et al. Intravenous infusion of L-isomers of phenylalanine and tryptophan stimulates gastric acid secretion at physiologic plasma concentrations in normal subjects and after parietal cell vagotomy. $f$ Clin Invest 1983;71:1254-62.

13 Niederau C, Sonnenberg A, Erckenbrecht J. Effects of intravenous infusion of amino acids, fat, or glucose on unstimulated pancreatic secretion in healthy humans. Dig Dis $S c i$ 1985;30:445-55.

14 Bursztein-De Myttenaere S, Gil KM, Heymsfield SB, et al. Gastric emptying in humans: influence of different regimens of parenteral nutrition. Am $\mathcal{F}$ Clin Nutr 1994;60: regimens

15 Marcus SN, Heaton KW. Intestinal transit, deoxycholic acid and the cholesterol saturation of bile: three inter-related factors. Gut 1986;27:550-8. 
16 Hussaini SH, Pereira SP, Veysey MJ, et al. Role of gallbladder in the pathogenesis of octreemptying and intestinal transit in the pathogenesis of oc
otide induced gallbladder stones. Gut 1996;38:775-83.

17 Bond JH, Levitt MD. Investigation of small bowel transit time in man utilising pulmonary hydrogen $\left(\mathrm{H}_{2}\right)$ measurements. F Lab Clin Med 1975;85:546-55.

18 Pijl H, Koppeschaar HPF, Cohen AF, et al. Evidence for brain serotonin-mediated control of carbohydrate consumption in normal weight and obese humans. Int $f$ Obes 1993;17:513-20.

19 Jansen JBMJ, Lamers CBHW. Radioimmunoassay of cholecystokinin in human tissue and plasma. Clin Chim Acta cystokinin in hu

20 Edelbroek M, Sun WM, Horowitz M, et al. Stereospecific effects of intraduodenal tryptophan on pyloric and duodenal motility in humans. Scand $\mathcal{F}$ Gastroenterol 1994;29: 1088-95.

21 Kerlin P, Zinsmeister AR, Phillips S. Relationship of motility to flow of contents in the human small intestine. Gastroenterology 1982;82:701-6.

22 Kellow JE, Borody TJ, Phillips SF, et al. Human interdigestive motility: variations in patterns from esophagus to colon. Gastroenterology 1986;91:386-95.

23 Sarna SK, Ammon HV, Walter LG, et al. Relationship between different phases of MMC and transit time: a reappraisal [abstract]. Gastroenterology 1985;88:A1570.

24 Bortolotti M, Barbara L. Interdigestive gastroduodenal motor activity in subjects with increased gastric acid secretion. Digestion 1988;41:156-60.

25 Yamamoto O, Matsunaga Y, Haga N, et al. Inhibition of phase III activity by acidifying stomach in vagally denervated and innervated dogs with gastric pouches. Gastroenterology 1994;106:1533-41.

26 Malfertheiner P, Domínguez-Muñoz JE. Effect of exogenous pancreatic enzymes on gastrointestinal and pancreatic hormone release and gastrointestinal motility. Digestion 1993;54(suppl 2):15-20.
27 Svenberg T, Nilsson I, Samuelson K, Welbourn RD. Studies on the causal relationship between gall-bladder emptying 520(suppl):59-61.

28 Qvist N, Øster-Jørgensen E, Pedersen SA, et al. Increases in plasma motilin follow each episode of gallbladder emptying during the interdigestive period, and changes in serum bile acid concentration correlate to plasma motilin. Scand $\mathcal{F}$ Gastroenterol 1995;30:122-7.

29 Vantrappen G, Janssens J, Peeters TL, et al. Motilin and the interdigestive migrating motor complex in man. Dig Dis Sci 1979;24:497-500

30 Nilsson I, Svenberg T, Hellström PM, et al. Pancreaticobilary juice releases motilin during phase I of the migrating motor complex in man. Scand 7 Gastroenterol 1993;28:80-4.

31 Penagini R, Misiewicz JJ, Frost PG. Effect of jejunal infusion of bile acids on small bowel transit and fasting jejunal motility in man. Gut 1988;29:789-94.

32 Masclee AMM, Beeren M, Rovati LC, et al. Effect of cholecystokinin on small intestinal motility and transit time in humans [abstract]. Gastroenterology 1994;106:A537.

33 Katschinski M, Schirra J, Beglinger C, et al. Intestinal phase of human antro-pyloro-duodenal motility: cholinergic and CCK-mediated regulation. Eur 7 Clin Invest 1996;26: 574-83.

34 Konturek JW, Thor P, Domschke W. Effect of nitric oxide on antral motility and gastric emptying in humans. Eur $\mathcal{f}$ Gastroenterol Hepatol 1995; 7:97-102.

35 Rodríguez-Membrilla A, Martínez V, Jiménez M, et al. Is nitric oxide the final mediator regulating the migrating myoelectric complex cycle? Am f Physiol 1995;31: G207-14.

36 Huizinga JD. The effect of amino acids on intestinal smooth muscle related to their content in blood and tissue. Eur F Pharmacol 1980;67:91-5.

37 Snape WJ Jr, Yoo S. Effect of amino acids on isolated colonic smooth muscle from the rabbit. $\mathcal{F}$ Pharmacol Exp Ther 1985;235:690-5. 